\title{
An evolving picture of the interactions between malaria parasites and their host erythrocytes
}

\author{
Thomas E Wellems ${ }^{1}$, Rick M Fairhurst ${ }^{1}$ \\ ${ }^{1}$ Laboratory of Malaria and Vector Research, National Institute of Allergy and Infectious Diseases, National Institutes of Health, \\ Bethesda, Maryland 20892-8132, USA \\ Cell Research (2012) 22:453-456. doi:10.1038/cr.2012.14; published online 24 January 2012
}

In patients with malaria, Plasmodium falciparum parasites multiply to enormous numbers in the bloodstream, initiating processes of erythrocyte destruction, endothelial activation and microvascular inflammation that cause devastating pathological effects on host tissues and organs. Recent research casts new light on a mechanism by which hemoglobin mutations may protect against these effects, and on a critical receptor-ligand interaction that provides fresh opportunities for the development of vaccines against blood-stage infection.

The symptoms of malaria occur in the period of the Plasmodium life cycle when erythrocytes are infested by parasites. This period commences when parasites emerge from the liver after replicating from the sporozoites introduced by a mosquito bite. The new blood-stage parasites multiply quickly as haploid, asexual forms in one-, twoor three-day cycles (depending on the Plasmodium species) and increase their numbers enormously, often infecting $1 \%$ or more of the trillions of erythrocytes in the bloodstream. These populations of asexual forms are required for the production of sexual gametocyte

Correspondence: Thomas E Wellems

E-mail: twellems@niaid.nih.gov forms that enter feeding mosquitoes, in which the parasites mate and produce new sporozoites for transmission to other human hosts. While large numbers of erythrocytes support the propagation and survival of malaria parasites, the inflammatory and erythrocyte-destroying effects of the parasite biomass cause devastating pathological effects on host tissues and organs.

These pathological effects and the deaths that they cause have exerted powerful selection pressure on the human genome over thousands of generations. Outcomes of this pressure include the sickle cell hemoglobin ( $\mathrm{HbS}$ ) mutation, which protects young children against the life-threatening complications of Plasmodium falciparum malaria, and various blood group antigens, which affect the ligand-receptor interactions utilized by different Plasmodium parasites for erythrocyte invasion. Two recent papers in Science and Nature offer fresh and interesting discoveries from research in these areas: (i) a molecular mechanism by which mutation of hemoglobin may protect against malaria [1], and (ii) a newly discovered ligandreceptor interaction that may be critical for $P$. falciparum invasion of human erythrocytes [2].

$\mathrm{HbS}$ represents a classic example of a balanced polymorphism: the heterozygous sickle cell trait (HbAS, from the inheritance of one normal $\mathrm{HbA}$ and one sickle $\mathrm{HbS} \beta$-globin-coding gene) protects against severe life-threatening malaria, while the homozygous HbAA condition offers no protection from malaria and the homozygous HbSS condition produces frequent fatalities from sickle cell anemia [3]. Although early studies implicated poor growth of $P$. falciparum in HbAS erythrocytes as a mechanism of protection that keeps parasitemias low, other work found that laboratory-adapted parasite clones grew normally in HbAS erythrocytes even under reduced oxygen conditions $[4,5]$. Further, it was clear that substantial parasitemias and frequent episodes of uncomplicated malaria occurred in HbAS as well as HbAA children, despite marked differences in the incidence of severe malaria between these groups [6]. Differential protection against severe malaria is also provided by hemoglobin $\mathrm{C}(\mathrm{HbC})$ in West Africa [7]. These field observations suggest a mechanism of protection that, instead of merely reducing the numbers of parasitized erythrocytes in the circulation, works to ameliorate the inflammation that arises at the host-parasite interface from the interactions of infected erythrocytes with the endothelium and other blood elements. Indeed, parasitized $\mathrm{HbAC}$ and HbAS erythrocytes show significant impairment of cytoadherence in association with perturbed display of the $P$. falciparum major cytoadherence 


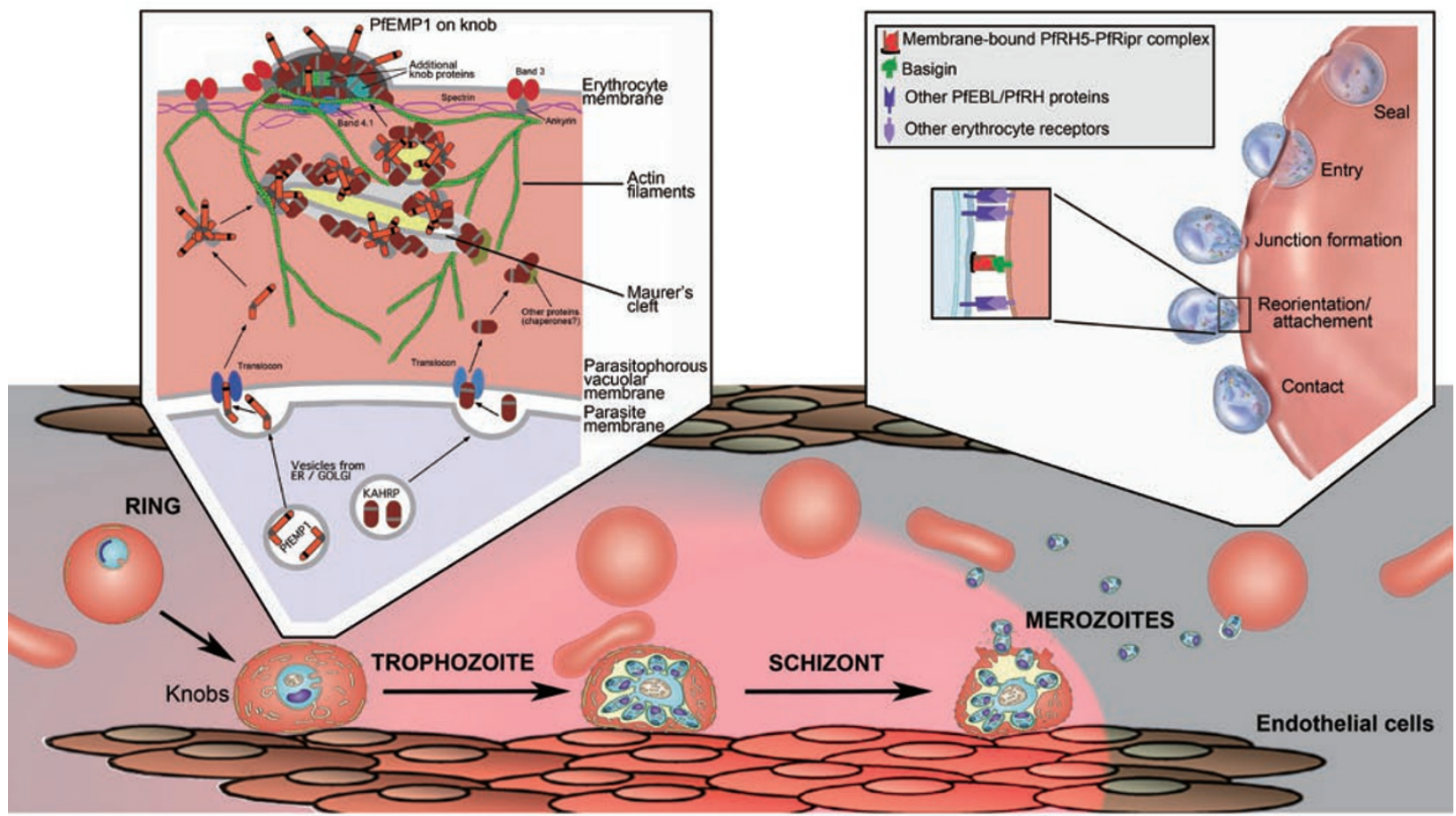

ENDOTHELIAL ACTIVATION \& INFLAMMATION

- Tissue factor display and thrombin activation

- Adhesion molecule upregulation

- von Willebrand Factor release and platelet adhesion

- Pro-inflammatory cytokine secretion

- Loss of endothelial barrier integrity

Figure 1 Junctures of pathogenesis in $P$. falciparum malaria: cytoadherence of parasitized erythrocytes to microvascular endothelium and parasite invasion of erythrocytes. P. falciparum parasites display knobs at the surface of their host erythrocytes as they mature from ring to trophozoite and schizont forms. PfEMP1 cytoadherence proteins are concentrated on knobs, where they bind receptors (e.g., CD36 and ICAM-1), activate endothelial cells, and recruit blood elements including platelets and white blood cells. By adhering in microvessels, the mature parasites avoid being carried by the bloodstream to the spleen where they are destroyed. Sequestration-related events lead to upregulation of tissue factor, resulting in thrombin and complement activation, platelet activation, cytokine production, endothelial dysfunction and inflammation [14]. To support the transport of PfEMP1 and other proteins including the knob-associated histidine-rich protein (KAHRP) to the erythrocyte membrane, $P$. falciparum parasites tether membranous Maurer's clefts beneath the cytoskeleton [9]. In knobs, PfEMP1 associates with KAHRP anchored to spectrin-actin-protein 4.1 complexes, to spectrin-actin junctions, and to the band 3-binding domain of ankyrin [15, 16]. Cryklaff et al. [1] report that $P$. falciparum remodels host actin into a network of filaments associated with Maurer's clefts and the erythrocyte membrane; this network may support protein and vesicle trafficking to the knobs. Hemoglobin variants $\mathrm{HbS}$ and $\mathrm{HbC}$ interfere with proper knob formation and PfEMP1 display, weaken the binding of parasitized erythrocytes to endothelium and may thereby reduce sequestration-related pathology [5, 8]. Aberrant Maurer's clefts and compromised remodeling of the actin network occur in $\mathrm{HbSC}$ and $\mathrm{HbCC}$ erythrocytes [1]; these abnormalities remain to be demonstrated in $\mathrm{HbAS}$ and $\mathrm{HbAC}$ erythrocytes. Inset (left) is adapted from ref [17]. Merozoites released from mature schizonts invade erythrocytes by steps of initial contact, reorientation with attachment, junction formation, and entry and membrane resealing. Most members of the PfEBL and $\mathrm{PfRH}$ protein families have overlapping and individually dispensable functions that support a diversity of invasion pathways by attachment to different blood group antigens. These events are followed by binding of $P$. falciparum AMA1 and RON proteins at the attachment interface, triggering junction formation (not shown) [18]. The interaction of the PfRH5-PfRipr complex [12] with basigin [2] appears to be essential for invasion and may have a critical function beyond the roles of binding and attachment that characterize other members of the PfEBL and PfRH families. Inset (right) is adapted from ref [18].

protein (PfEMP1) on abnormal knob protrusions $[5,8]$.
Using cryo-electron tomography, Cryklaff et al. [1] show that P. falci- parum hijacks and remodels erythrocyte actin into a network that may support 
the trafficking of PfEMP1 and other virulence proteins to the host cell membrane, where these proteins form the knobs involved in sequestration-related events and inflammation (Figure 1). Branching patterns of actin were found in association with Maurer's clefts, membranous compartments that have an important role in exporting proteins from the parasite to the periphery of the host cell cytoplasm [9]. Further, Cryklaff et al. [1] observed significantly reduced actin remodeling and aberrant Maurer's clefts in $\mathrm{HbCC}$ and $\mathrm{HbSC}$ erythrocytes, suggesting that these mutant hemoglobin states may interfere with the installation of actin scaffolds that help to tether Maurer's clefts and support vesicle and protein trafficking to the erythrocyte membrane.

Are there also significant differences between the actin networks and/ or Maurer's clefts of $\mathrm{HbAS}, \mathrm{HbAC}$ and HbAA erythrocytes? HbAS and HbAC are, after all, the prevalent malariaprotective states of $\mathrm{HbS}$ and $\mathrm{HbC}$. On this question the report of Cryklaff et $a l$. [1] is unfortunately silent; actin remodeling and Maurer's clefts in HbAS and $\mathrm{HbAC}$ erythrocytes still need to be investigated, as do the effects of $\mathrm{HbAS}$ and $\mathrm{HbAC}$ erythrocyte extracts on actin polymerization in vitro. Mechanisms apart from disturbed actin remodeling may still account for abnormal PfEMP1 display and knob formation on parasitized HbAC and HbAS erythrocytes. For example, hemichromes generated more readily from oxidation of $\mathrm{HbC}$ or $\mathrm{HbS}$ may physically hinder the docking of virulence proteins and thus interrupt the formation of regular knob arrays in the erythrocyte cytoskeleton (where hemichromes are known to bind). Another possibility is that $\mathrm{HbC}$ and $\mathrm{HbS}$ elevate the levels of oxidative stress in parasitized erythrocytes, damaging membranes and biochemically hampering the functions of Maurer's clefts and their associated vesicles in the transport of knob-forming proteins. The challenge for the research field in the coming years will be to sort out these possible mechanisms and their contributions to malaria protection by the heterozygous $\mathrm{HbAS}$ and $\mathrm{HbAC}$ conditions.

$P$. falciparum invades erythrocytes by various pathways involving different ligand-receptor interactions [10]. Partner ligands in these interactions include members of two molecular families known as the EBA (erythrocyte binding antigen) and RBL (reticulocyte bindinglike) proteins. In P. falciparum, members of these families are termed PfEBL (P. falciparum erythrocyte binding-like) and PfRH (P. falciparum reticulocyte binding-like homolog) proteins. Evidence suggests that the roles of many of these proteins are overlapping: $P$. falciparum lines can often invade erythrocytes that lack receptors for specific PfEBL or PfRH proteins (because of inherent mutations or enzyme treatment) and, conversely, knockout parasites that do not express individual PfEBL or PfRH proteins have been found to efficiently invade erythrocytes. It thus came as a surprise that focused efforts to knock out the gene encoding an atypical, foreshortened member of the PfRH family, PfRH5, did not succeed in two laboratories $[10,11]$. While polymorphisms in PfRH5 could be linked to receptor preferences and an ability of a P. falciparum line to also invade Aotus monkey erythrocytes [11], the function of PfRH5 as a parasite ligand and the erythrocyte receptor(s) that it uses for invasion remained unknown.

Crosnier et al. [2] have now identified the PfRH5 receptor by applying an avidity-based extracellular interaction screen (AVEXIS) to the expressed proteins of an erythrocyte ectodomain library. Results show that PfRH5 binds an isoform of basigin on erythrocytes (BSG-S, the Ok blood group antigen, CD147) and that P. falciparum appears to generally require this interaction to efficiently invade human erythrocytes. In contrast to the effects of glycan removal (by neuraminidase) from Aotus erythrocytes on PfRH5 binding and parasite in- vasion by particular P. falciparum lines [11], removal of all glycans from human basigin does not alter PfRH5 binding [2]. Further, in experiments with multiple P. falciparum clones, Crosnier et al. show that the invasion of human erythrocytes is potently inhibited by a soluble pentamerized form of basigin, by anti-basigin monoclonal antibodies, and by reduction of basigin levels on the erythrocyte surface [2].

Two of five single amino acid polymorphisms (E92K, L90P) in basigin affected PfRH5 binding to or invasion of human erythrocytes. While one of these polymorphisms (E92K) is associated with a relatively rare $\mathrm{Ok}^{\mathrm{a}-}$ blood group in Japan, there are no data to suggest that L90P (or perhaps another undiscovered basigin polymorphism) was naturally selected to high prevalence in malaria-endemic areas. Although the native function of basigin on human erythrocytes is unknown, its high level of conservation suggests that $P$. falciparum may have evolved to depend on basigin to a much greater extent than on other, more polymorphic receptors. Interestingly, PfRH5 is also unlike other members of the PfEBL and PfRH families in that it lacks a transmembrane domain; recent evidence indicates that it forms a complex with a processed EGFlike PfRH5-interacting protein (PfRipr) and that this complex, in turn, associates tightly with another partner on the merozoite membrane [12] (Figure 1). Attempts to disrupt the PfRipr gene also have been unsuccessful [12].

These findings suggest a critical role for the PfRH5-PfRipr complex that goes beyond an additional contribution of mechanical attachment within the binding repertoire of PfEBL and PfRH proteins. Possibilities for essential function might include PfRH5-PfRipr participation in a signaling pathway required for invasion, or an essential partnership in the junction during merozoite entry (Figure 1). Recent results also show promise for the use of PfRH5 and PfRipr as targets for intervention: rabbit IgG antibodies 
against virally-vectored, full-length PfRH5 outperformed antibodies that were generated by the same strategy against nine other erythrocytic-stage vaccine candidates, including four other PfEBL and PfRH proteins [13]; rabbit antibodies against PfRipr likewise inhibited merozoite attachment and parasite growth in culture [12]. Although variations in the susceptibility of different $P$. falciparum lines were observed in these studies (likely due to different utilization patterns of ligand-receptor interactions), striking neutralization was nevertheless achieved in all cases. These results support PfRH5 and other components of its binding complex as new targets for therapeutic intervention and give an important boost to vaccine efforts against the asexual blood stages of P. falciparum.

\section{References}

1 Cyrklaff M, Sanchez CP, Kilian N, et al. Hemoglobins $\mathrm{S}$ and $\mathrm{C}$ interfere with actin remodeling in Plasmodium falciparum-infected erythrocytes. Science 2011; 334:1283-1286.

2 Crosnier C, Bustamante LY, Bartholdson $\mathrm{SJ}$, et al. Basigin is a receptor essential for erythrocyte invasion by Plasmodium falciparum. Nature 2011; 480:534-537.

3 Allison AC. Polymorphism and natural selection in human populations. Cold
Spring Harb Symp Quant Biol 1964; 29:137-149.

4 Becker K, Tilley L, Vennerstrom JL, et al. Oxidative stress in malaria parasiteinfected erythrocytes: host-parasite interactions. Int $J$ Parasitol 2004; 34:163-189.

5 Cholera R, Brittain NJ, Gillrie MR, et al. Impaired cytoadherence of Plasmodium falciparum-infected erythrocytes containing sickle hemoglobin. Proc Natl Acad Sci USA 2008; 105:991996.

6 Williams TN, Mwangi TW, Wambua $\mathrm{S}$, et al. Sickle cell trait and the risk of Plasmodium falciparum malaria and other childhood diseases. $J$ Infect Dis 2005; 192:178-186.

7 May J, Evans JA, Timmann C, et al. Hemoglobin variants and disease manifestations in severe falciparum malaria. JAMA 2007; 297:2220-2226.

8 Fairhurst RM, Baruch DI, Brittain NJ, et al. Abnormal PfEMP1 display on hemoglobin $\mathrm{C}$ erythrocytes may protect against malaria. Nature 2005; 435:1117-1121.

9 Hanssen E, Sougrat R, Frankland S, et al. Electron tomography of the Maurer's cleft organelles of Plasmodium falciparum-infected erythrocytes reveals novel structural features. $\mathrm{Mol} \mathrm{Mi-}$ crobiol 2008; 67:703-718.

10 Cowman AF, Crabb BS. Invasion of red blood cells by malaria parasites. Cell 2006; 124:755-766.

11 Hayton K, Gaur D, Liu A, et al. Erythrocyte binding protein PfRH5 polymorphisms determine species-specific pathways of Plasmodium falciparum invasion. Cell Host Microbe 2008; 4:40-51.

12 Chen L, Lopaticki S, Riglar DT, et al. An EGF-like protein forms a complex with PfRh5 and is required for invasion of human erythrocytes by Plasmodium falciparum. PLoS Pathog 2011; 7:e1002199.

13 Douglas AD, Williams AR, Illingworth $\mathrm{JJ}$, et al. The blood-stage malaria antigen PfRH5 is susceptible to vaccineinducible cross-strain neutralizing antibody. Nat Commun 2011; 2:601.

14 Francischetti IM, Seydel KB, Monteiro RQ. Blood coagulation, inflammation, and malaria. Microcirculation 2008; 15:81-107.

15 Oh SS, Voigt S, Fisher D, et al. Plasmodium falciparum erythrocyte membrane protein 1 is anchored to the actinspectrin junction and knob-associated histidine-rich protein in the erythrocyte skeleton. Mol Biochem Parasitol 2000; 108:237-247.

16 Magowan C, Nunomura W, Waller KL, et al. Plasmodium falciparum histidinerich protein 1 associates with the band 3 binding domain of ankyrin in the infected red cell membrane. Biochim Biophys Acta 2000; 1502:461-470.

17 Papakrivos J, Wellems TE. Designer transport of malaria proteins in erythrocytes. Blood 2005; 105:3757-3758.

18 Srinivasan P, Beatty WL, Diouf A, et al. Binding of Plasmodium merozoite proteins RON2 and AMA1 triggers commitment to invasion. Proc Natl Acad Sci USA 2011; 108:13275-13280. 